\title{
The Configural Processing of Facial Expression in Identification of Facial Parts: Are Eyes More Eloquent Than Lips?
}

\author{
Sayako Ueda \\ Japan Women’s University, Kawasaki, Japan
}

\begin{abstract}
We investigated the role of configural processing of facial expression in identification of single facial features and considered whether configural processing differentially affects people's ability to identify certain expressive facial features (eyes vs. mouth). Two stimulus types were created by embedding different expressive facial features within a facial image to produce expressively incongruent images (e.g., happy face and surprised mouth) and expressively congruent ones (e.g., happy face and happy mouth). Participants had to pattern match shapes of different facial features (eyes and mouth). Results from a series of experiments involving upright, inverted and photo-negative faces indicated that identification of the local facial features in incongruent condition is more difficult than that in congruent condition; this effect is stronger in identification of the eyes than in identification of the mouth. These findings suggested a significant role for holistic configural processing of expression in the identification of a facial feature, and the eyes were not more informative than the mouth.
\end{abstract}

Keywords: configural processing, facial expression, facial parts

\section{Introduction}

The human face conveys a great variety of information, such as an individual's identity, age, gender, emotional state, race, physical attractiveness and even one's volitional state. Adults can detect and interpret these important social cues at a glance. Their expertise in facial recognition has been attributed to configural processing. The term "configural processing” refers to processing not just the shapes of individual's facial features but also the relations among them, and it is contrasted with the term "featural processing", which refers to sensitivity to differences among faces in the appearance of individual features. Maurer and colleagues' review (Maurer, Le Grand, \& Mondloch, 2002) illustrated that configural processing can be divided into several levels, based on the findings of the developmental studies that these processing mature at different rates. One form that shows an early developmental onset is holistic processing (Carey \& Diamond, 1994; Tanaka, Kay, Grinnell, Stansfield, \& Szechter, 1998), the processing facial structures as a single gestalt. And a second form that shows increasing proficiency with age is second-order relational processing (Freire \& Lee, 2001; Mondloch, Le Grand, \& Maurer, 2002), sensitivity to the spatial relationships among individual's features in a face. Studies addressing these configural processing have generally concentrated on facial identity.

The current consensus in research on the recognition of facial identity is that configural processing is particularly important for facial recognition. There are four convincing demonstrations of configural processing based, respectively, upon the inversion effect, the composite face effect, the part-whole recognition effect and

Sayako Ueda, Ph.D., Faculty of Integrated Arts and Social Science, Japan Women’s University. 
the negation effect. The inversion effect, in which accuracy of facial identity is impaired when faces are upside down (Yin, 1969), is caused largely by disruptions in holistic and second-order relational processing (Barton, Keenan, \& Bass, 2001; Barton, Depak, \& Malik, 2003; Collishaw \& Hole, 2000; Freire, Lee, \& Symons, 2000; Leder \& Bruce, 2000; Le Grand, Mondloch, Maurer, \& Brent, 2001; Mondloch et al., 2002; Rhodes, Brake, \& Atkinson, 1993; Searcy \& Bartlett, 1996). However, inverting has an insignificant effect on featural processing (Barton et al., 2001; Freire et al., 2000; Leder \& Bruce, 1998, 2000; Leder, Candrian, Huber, \& Bruce, 2001; Le Grand et al., 2001). The composite face effect, in which adults are slower and less accurate in recognizing the top half of one face presented in a composite with bottom half of another face than when the two halves are offset laterally (Young, Hellawell, \& Hay, 1987), is caused by disruptions in holistic processing (Carey \& Diamond, 1994; Hole, 1994; Hole, George, \& Dunsmore, 1999; Le Grand et al., 2004; Michel, Rossion, Han, Chung, \& Caldara, 2006). Disruptions in holistic process also cause the part-whole recognition effect. In this case, adults are more accurate in recognizing a specific feature from an individual's face (e.g., Larry's nose) when it is presented in the context of the whole face (e.g., Larry's nose in Larry's face) than when presented in isolation (Farah, Tanaka, \& Drain, 1995; Tanaka \& Farah, 1993; Tanaka \& Sengco, 1997). Finally, the negation effect, in which faces are particularly difficult to recognize in photographic negatives (Galper, 1970; Galper \& Hochberg, 1971), is caused by disruptions in second-order relational processing (Hayes, Morrone, \& Burr, 1986; Hole, George, \& Dunsmore, 1999; Kemp, McManus, \& Pigott, 1990; Kemp, Pike, White, \& Musselman, 1996; Lewis \& Johnston, 1997; White, 2001).

In addition to recognizing people's facial identities, we can infer emotional states from facial expressions. One might assume that facial expressions are processed in a feature-based manner, wherein emotions like surprise are expressed in the form of a localized feature, such as an open mouth or raised eyebrows. However, little is known about the role of configural information in recognizing facial expressions, several studies have demonstrated that configural cues appear to play a significant role in recognizing expressions. Calder, Young, Keane, and Dean (2000) discovered that the composite face effect in emotion recognition is similar to a composite effect in facial recognition. Composite expressions are produced by aligning the top half of a face expressing one emotion (e.g., anger) with the bottom half of another expressing another emotion (e.g., happiness). Calder found that recognition of the emotion shown in one half is slower and less accurate than when the two halves are offset laterally (Calder \& Jansen, 2005; Durand, Gallay, Seigneuri, Robichon, \& Baudouin, 2007; White, 2000). White (1999) demonstrated the presence of an inversion effect in the recognition of emotion; the participant's judgment of facial expressions was impaired when faces were inverted than upright (Durand et al., 2007; Fallshore \& Bartholow, 2003).

Calder et al. (2000) revealed that there are subtle differences in configural processing for identity and expression (Calder \& Jansen, 2005). However, there is also some evidence of an interaction between the processing of identity and expression (Gallegos \& Tranel, 2005; Ganel, 2005; Kaufmann \& Schweinberger, 2004; Lander \& Metcalfe, 2007; Posamentier \& Abdi, 2003; Schweinberger \& Soukup, 1998; Wild-Wall, Dimigen, \& Sommer, 2008).

Given the above observations, one goal of the present research is to ascertain if the configural processing involved recognizing facial expression may also play a role in one's ability to identify faces; conversely, it is possible that the configural processing involved in identification processing may have some impact upon a processing of facial expression. In the present study, we investigated whether the configural processing of expression has an influence on the identification of a single facial feature (e.g., the pattern-matching of mouth 
in a smiling face). If face expresses an emotion, this may affect how a viewer identifies a specific facial feature.

A second goal is to investigate whether configural processing of facial expressions exerts a selective impact on the identification of certain facial features. That is, we considered whether or not either the eyes or mouth were particularly influenced by configural processing of facial expression. Eyes are often accorded a special role according to folklore, "Eyes are the windows to the soul" and "Eyes are more eloquent than lips". Baron-Cohen (1995) proposed the existence of the "language of the eyes", in which the presentation of the eye region alone gives cues of a person's mental state (Baron-Cohen, Wheelwright, \& Joliffe, 1997). If eyes alone are more telling than the mouth as folklore claims, i.e., the eyes have cues that play an important role in facial perception, we would expect that the identification of the eyes would be less influenced by the configural processing of facial expressions than the mouth.

In the present series of experiments, we created two types of stimulus materials involving, respectively, incongruent and congruent emotional expressions. In both, features of eyes and mouth were of interest. This manipulation described details in Figure 1 was slightly different from manipulations that the preceding studies demonstrated. Therefore, incongruent faces, i.e., faces with incongruent emotional expressions, were prepared by transferring a feature in one face where it expresses a congruent emotion (e.g., happy eyes in a face expressing happiness) to another face that originally expressed a different emotion (e.g., happy eyes in a face expressing fear). On the other hand, congruent faces did not undergo any kind of feature replacement, a given feature and face expressed the same emotion (e.g., happy eyes in a happy face). Examples of such faces are shown in Figure 1. One issue arose in this situation involves the possibility that some expressions of the eyes would be easier to pattern-match for shapes at times (recognizable eyes) and that some of the mouths would be easier to pattern-match for shapes at other times (recognizable mouth). Because we aimed to investigate whether the influence of configural processing of facial expressions on the identity of single facial feature expressions, it was necessary to compare facial features with the same recognizability. Consequently, we conducted a preliminary experiment to identify recognizable eyes and mouths. Then, these were used when replacing in incongruent faces.

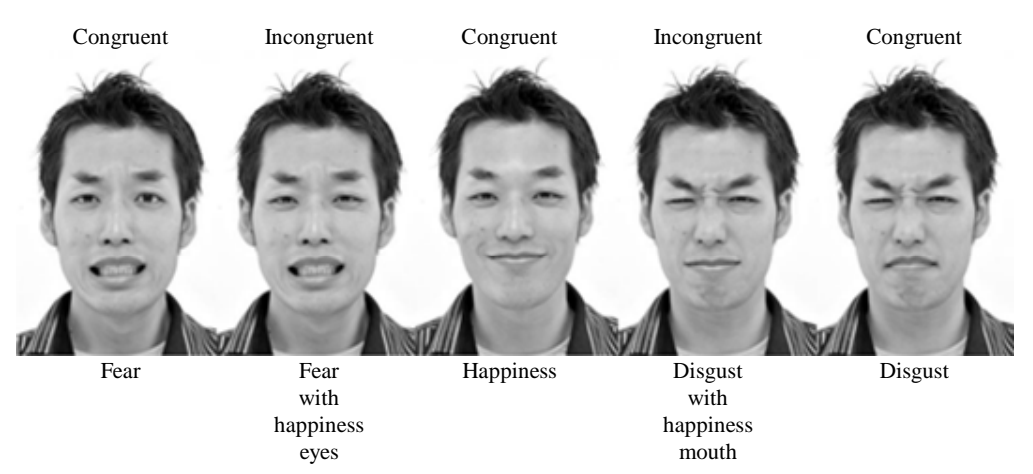

Figure 1. Examples of the stimulus used in Experiment.

Experiment 1 examined the possible role of configural processing of expressions in viewers' identification of a single facial feature and also investigated the possibility that this configural processing may differ depending upon the facial features, i.e., eyes vs. mouth. The index of the role of configural processing of expression in facial feature identification was based on whether the pattern-matching of a single facial feature in incongruent faces was less accurate than that in congruent faces. 
Experiment 2 used the paradigm of Experiment 1, with the exception that the images were presented in an inverted orientation, which enabled us to ascertain if the role of configural processing of expression in facial feature identification involved holistic and/or second-order relational processing. The determination has been based on whether evidence of configural processing, as shown in Experiment 1, was impaired in the inversion condition (Experiment 2). If such impair was observed, disruption of holistic and/or second-order processing should lead to poor difference of feature identification accuracy between incongruent and congruent faces.

Experiment 3 continued with the Experiment 1 paradigm but stimuli in this experiment involved photographic negative transforms of the original faces. We investigated whether the role of configural processing of expression in identification of a single facial part was resistant to the negation effect. This enabled us to ascertain if the role of configural processing of expression in facial feature identification uninvolved second-order relational processing. If such resistance was observed in the negation condition (Experiment 3), only holistic processing should lead to poorer feature identification accuracy in incongruent than in congruent in Experiment 1.

\section{Preliminary Experiment}

In this section, the aim was to identify eyes and mouths that were reliably recognized in order to create effective incongruent faces for Experiment 1.

\section{Method}

Participants. Ten Japanese females, aged between 21 and 25 years, participated in the experiment for payment. All the participants were right-handed and had normal or corrected-to-normal vision.

Materials. The stimuli were prepared from color photographs of six Japanese models ( 3 males, 3 females) taken at a photography studio in 2009. All models consented to use of their photographs in the experimental study. The faces were photographed from the front, and each model assumed each of six facial expressions, namely, happiness, sadness, anger, fear, disgust and surprise, which resulted in a total of 36 faces (i.e., 6 models $\times 6$ expressions). The faces were scaled standardizing the width of all the faces at the pupil level into 300 pixels.

Design. The experiment involved a 6 (expression) $\times 2$ (task) within-subjects factorial design. The two task types were eyes-identification task and the mouth-identification task. The dependent variable was the correct proportions.

Procedure. There were 6 (expressions) $\times 2$ (task types) $\times 6$ (models) $=72$ trials in the experiment. Each trial began with a 500-ms visual cue (cursor), presented in a randomly selected location to alert the participants as to where a target face would be displayed. This was followed by a single (target) face for $200 \mathrm{~ms}$. The target was followed by a $500 \mathrm{~ms}$ inter-stimulus interval (black screen). Next, a set of six different single feature images appeared (eyes only or mouth only); these feature images were extracted from photographs of the facial model used in the immediately preceding target image. Each feature image expressed one of six emotions (happiness, sadness, anger, fear, disgust and surprise), and only one feature image was a correct match to preceding target's feature. For each trial, the target face and test images were prepared from the photographs of the same model. Positions of test images were counterbalanced across the participants. The participants saw each target stimulus once, with the order of the 72 stimuli randomized.

Participants were instructed to identify, as quickly as possible, the facial feature used in the target face by 
clicking the mouse on the test image that matched the target. When the participants had made their decision, the experiment proceeded to the next trial. Each task type (eye identification, mouth-identification) occurred on 36 of the 72 trials, with order of presentation randomized.

\section{Results}

Their correct proportions arcsin-transformed and submitted to a two-factor repeated measures ANOVA (analysis of variance) that crossed six levels of expression (happiness, sadness, anger, fear, disgust and surprise) with two types of task (eye-identification and mouth-identification). A significant main effect of expressions $\left(F_{(5,45)}=8.19, M S E=274.95, p<0.01\right)$ appeared, but the main effect of task was not significant $(F<1)$. However, the interaction of expression with task was significant $\left(F_{(5,45)}=10.82, M S E=295.52, p<0.01\right)$. Post-hoc comparisons of performance on expressions within each task type showed that happiness, surprise and disgust were significantly more identifiable than fear ( $p<0.01$, Bonferroni corrected) in the eye-identification task, whereas happiness, surprise and fear were significantly more identifiable than anger $(p<0.01$, Bonferroni corrected) in the mouth-identification task. These results revealed that happiness, surprise and disgust were easier to pattern-match from the single pair-of-eyes feature of the face (recognizable-eyes expressions), whereas, happiness, surprise and fear were easier to pattern-match from the single mouth feature of the face (recognizable-mouth expressions). In Experiment 1, only the more recognizable pair-of-eyes and the more recognizable mouths were used to create the incongruent faces.

\section{Experiment 1}

In Experiment 1, incongruent faces and congruent faces were used as stimuli to investigate the possible role of configural processing of expression in identifying single facial features and the possible differences in its influence on specific feature identifications of eyes versus mouth. If the participants' pattern-matching of single facial feature in incongruent faces is less accurate than that in congruent faces, then the configural processing of expression should play an important role in the identification of the single facial feature. However, if the identification of the eyes is less influenced by the configural processing of expression than the identification of the mouth, then the eyes are expected to be more expressive than the mouth, as the folklore claims (i.e., eyes have cues that play important roles in facial perception).

\section{Method}

Participants. Sixteen Japanese females aged between 19 and 45 years participated in the experiment for payment. All but one participant were right-handed and all had normal or corrected-to-normal vision.

Materials. The stimuli were the same as those in the preliminary experiment except that each model expressed four emotions, happiness, fear, disgust and surprise, which had been selected in the previous experiment. Incongruent and congruent faces were then created from these facial expressions. The incongruent faces were prepared by replacing a single facial feature from a face with one that expressed each feature's recognizable emotions (i.e., if the single feature that was replaced was the eyes, then the emotions of happiness, disgust and surprise were used; however, when the replaced single feature was the mouth, then the emotions of happiness, fear and surprise were used) with the same single feature that expressed emotions that differed from another face's recognizable emotions, i.e., if the replaced feature was the eyes, then happiness, fear and surprise were used; however, if the replaced single feature was the mouth, then happiness, disgust and surprise were used. The single feature and face of each incongruent face were always that of same model, which resulted in 
seven possible eyes/face combinations (happiness/fear, happiness/surprise, disgust/happiness, disgust/fear, disgust/surprise, surprise/happiness and surprise/fear) and seven possible mouth/face combinations (happiness/disgust, happiness/surprise, fear/happiness, fear/disgust, fear/surprise, surprise/happiness and surprise/disgust). A total of 84 different incongruent faces (i.e., 6 models $\times 14$ combinations) was generated. Congruent faces were the ones in which the single features were not replaced. This gave three possible eyes/face combinations (happiness/happiness, disgust/disgust and surprise/surprise) and three possible mouth/face combinations (happiness/happiness, fear/fear and surprise/surprise); as a result, a total of 36 congruent faces (i.e., 6 models $\times 6$ combinations) were formed.

Design. The experimental design crossed stimulus congruence (congruent and incongruent) with task (eye-identification and mouth identification) in a two-factor repeated measures design, which resulted in four different trial formats: incongruent eyes, incongruent mouth, congruent eyes and congruent mouth. Two dependent variables were proportion correct responses and correct reaction times.

Procedure. The procedure resembled that of the preliminary experiment. The only difference was that while the target faces in this experiment were either incongruent faces or congruent faces. The set of test images with single features was the same as that of the preliminary experiment (i.e., happiness, sadness, anger, fear, disgust and surprise). The trial formats were as follows: the incongruent eyes format (42), the incongruent mouth format (42), the congruent eyes format (18) and the congruent mouth format (18). Four formats were randomly intermixed within the experiment. As a result, the experiment encompassed a total of 120 trials (42+ $42+18+18=120)$.

\section{Results}

The participants' untransformed correct proportions and mean correct reaction times are summarized in Figure 2 (horizontal line indicates chance level responding).

Correct proportions. The participants' correct proportions were arcsin-transformed and submitted to a two-factor repeated measures ANOVA investigating stimulus congruence (incongruent face and congruent face; repeated measures) and task (eyes-identification and mouth-identification). There were significant main effects of congruence $\left(F_{(1,15)}=36.96, M S E=177.56, p<0.01\right)$, task $\left(F_{(1,15)}=62.81, M S E=27.02, p<0.01\right)$; a significant interaction between congruence and task $\left(F_{(1,15)}=57.17, M S E=16.39, p<0.01\right)$ was also observed. Post-hoc comparisons of performance on the stimulus congruence within each task showed significant differences ( $p<0.01$, Bonferroni corrected) between the incongruent and congruent conditions (incongruent correct < congruent correct). Post-hoc comparisons of performance on task within each level of congruence revealed a significant difference ( $p<0.05$, Bonferroni corrected) between the eyes and mouth conditions only for the incongruent condition (eyes correct $<$ mouth correct).

Reaction times. A two-factor repeated measures ANOVA investigating the same two factors (congruence and task) was performed on the remaining mean correct RTs (Reaction times). There was significant main effect of congruence $\left(F_{(1,15)}=17.10, M S E=4.14, p<0.01\right.$; incongruent RTs $>$ congruent RTs), task $\left(F_{(1,15)}=\right.$ 8.46, $M S E=2.95, p<0.05$; eyes RTs $>$ mouth RTs) was observed. Furthermore, no significant interaction was observed between stimulus type and task type $(F<1)$.

\section{Discussion}

The results of Experiment 1 showed that the configural processing of expression played an important role in the identification of a single facial feature. Participants were significantly less accurate in identifying single facial 
features in incongruent conditions than that in congruent conditions. Moreover, the strength of this effect differed when the participants were asked to identify the eyes from that when they were asked to identify the mouth, eyes were more difficult to identify than the mouth. Despite of this, in the congruent condition identifications of the two single features were equivalent and well above chance. Contrary to our expectations, eyes were less informative than the mouth. Reaction-time analyses complemented the patterns of accuracy found in Experiment 1. Reaction times were significantly slower in incongruent conditions than that in congruent conditions, and the eye-identification task took participants longer time than the mouth-identification task.
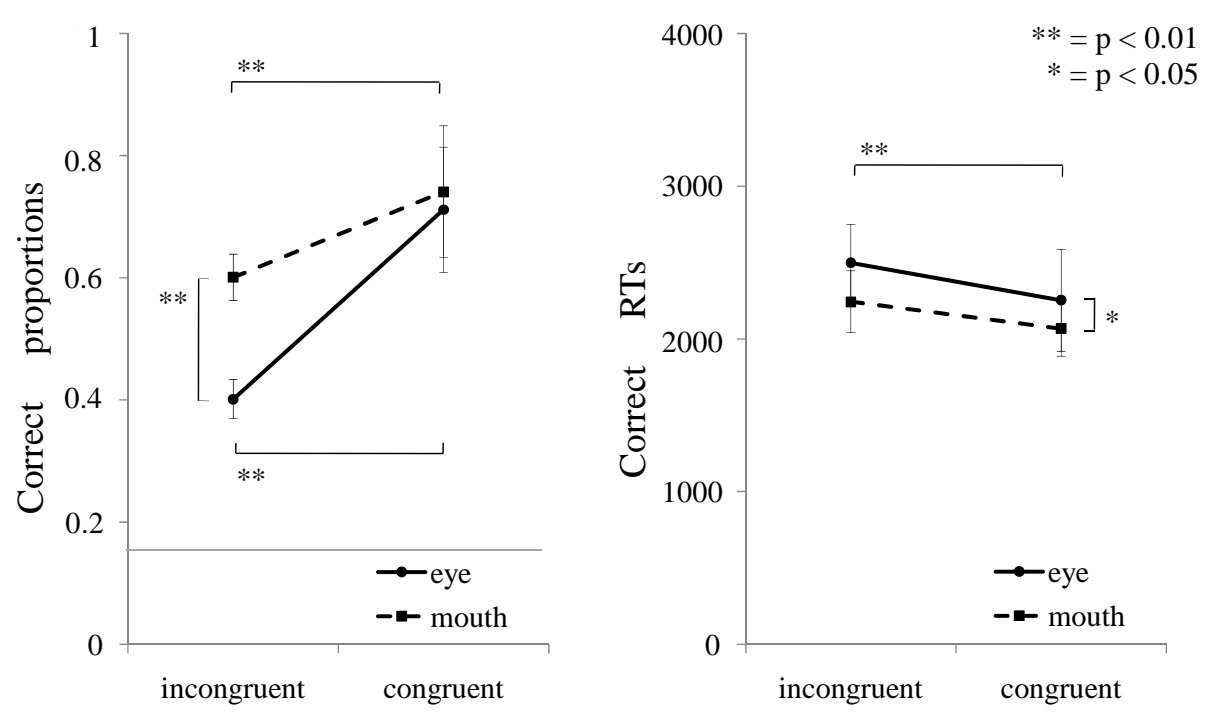

Figure 2. Mean correct proportions and RTs in Experiment 1. Error bars depict standard deviation. Overall, participants were significantly less accurate in identifying a single facial feature in the incongruent condition than in the congruent condition. Within incongruent condition, eyes were more difficult to identify than the mouth. Moreover, RTs in incongruent condition were slower than in congruent condition.

Configural processing has been differentiated into holistic processing and second-order relational processing. However, the type of processing occurring in Experiment 1 was unclear, because our paradigm using in Experiment 1 was slightly different from manipulations that the preceding studies demonstrated. Therefore, in Experiments 2 and 3, we further explored whether configural information played an important role in pattern-matching single facial feature. If disruption of feature identification accuracy in incongruent conditions was not observed in inverted facial images but in negation facial images, the configural processing showed in Experiment 1 would be only holistic processing.

\section{Experiment 2}

Experiment 2 used the paradigm of Experiment 1, with the exception that all images were presented in an inverted orientation which enabled us to address whether the role of configural processing of expression in identifying a single facial feature was likely to reflect holistic and second-order relational processing. The index of holistic and second-order relational processing was determined on the basis of whether the evidence of configural processing shown in Experiment 1 was impaired in the inversion condition (in Experiment 2). Specifically, if holistic and second-order relational processing of expression was disrupted by inverted facial images, viewers' judgment of identifying a single facial feature should be tied largely to one dimension of facial processing, featural processing. In that case, poor difference between the accuracy in incongruent and 
that in congruent would be observed, and RTs in incongruent conditions would not be slower than that in congruent conditions.

\section{Method}

Participants. Sixteen Japanese females aged between 20 and 33 years participated in the experiment for payment. All the participants were right-handed and had normal or corrected-to-normal vision.

Materials. The stimuli were the same as those in Experiment 1 except that all images (target and test) were presented in an inverted orientation.

Design. The design was identical to that of Experiment 1. This included the repeated measures factors, stimulus congruent (incongruent and congruent) and task (eye-identification and mouth-identification). The four different trial formats were as follows: incongruent eyes, incongruent mouth, congruent eyes and congruent mouth formats. Again, dependent variables were proportion correct identifications and correct reaction times.

Procedure. The procedure was identical to that of Experiment 1.

\section{Results}

The participants' untransformed correct proportions and mean correct reaction times were summarized in Figure 3 (horizontal line indicates chance level responding).

Correct proportions. A two-factor repeated measures ANOVA investigating the same two factors defined in Experiment 1 (i.e., stimulus congruence and task) was performed on the arcsin-transformed correct proportions. There were significant main effects of stimulus congruence $\left(F_{(1,15)}=6.02, M S E=98.19, p<0.05\right)$ task $\left(F_{(1,15)}=17.59, M S E=21.79, p<0.01\right)$, and a significant interaction between congruence and task $\left(F_{(1,15)}\right.$ $=19.27, M S E=20.21, p<0.01$ ) was observed. Post-hoc comparisons of performance on congruence within the identify-eyes task showed significant differences $(p<0.01$, Bonferroni corrected) between incongruent and congruent conditions (incongruent corrects $<$ congruent corrects), but no significant difference was observed in the case of the mouth-identification task (incongruent corrects = congruent corrects). Post-hoc comparisons of performance on task within each stimulus congruence showed that there was a significant difference $(p<0.01$, Bonferroni corrected) between the eyes and mouth expressions only for the incongruent condition (eyes corrects $<$ mouth corrects).

Reaction times. A two-factor repeated measures ANOVA investigating the same two factors defined in Experiment 1 (i.e., congruence and task) was performed on the remaining mean correct RTs. There was significant main effect of stimulus congruence $\left(F_{(1,15)}=16.14, \operatorname{MSE}=2.53, p<0.01\right.$; congruent RTs $>$ incongruent RTs $)$ and task $\left(F_{(1,15)}=15.09\right.$, MSE $=17.92, p<0.01$; eyes RTs $>$ mouth RTs $)$ was observed. No significant interaction between congruence and task $(F<1)$ was observed.

\section{Discussion}

The results of Experiment 2 showed that accuracy difference between incongruent and congruent conditions shown in Experiment 1 was not observed in the mouth-identification condition, and slower RTs in incongruent condition than that in congruent condition were not observed. These results suggested that inverting images significantly disrupted the role of configural processing of expression in identifying mouth. Other, within eye-identification condition, participants' accuracy showed no difference between the incongruent and congruent conditions. One possible explanation for this poor inversion effect in the eye-identification condition was that the influence of configural processing of expression on the 
pattern-matching of single facial part was much stronger in the eyes than that in the mouth. Finally, these results indicated that the inversion effect was displayed in Experiment 2.
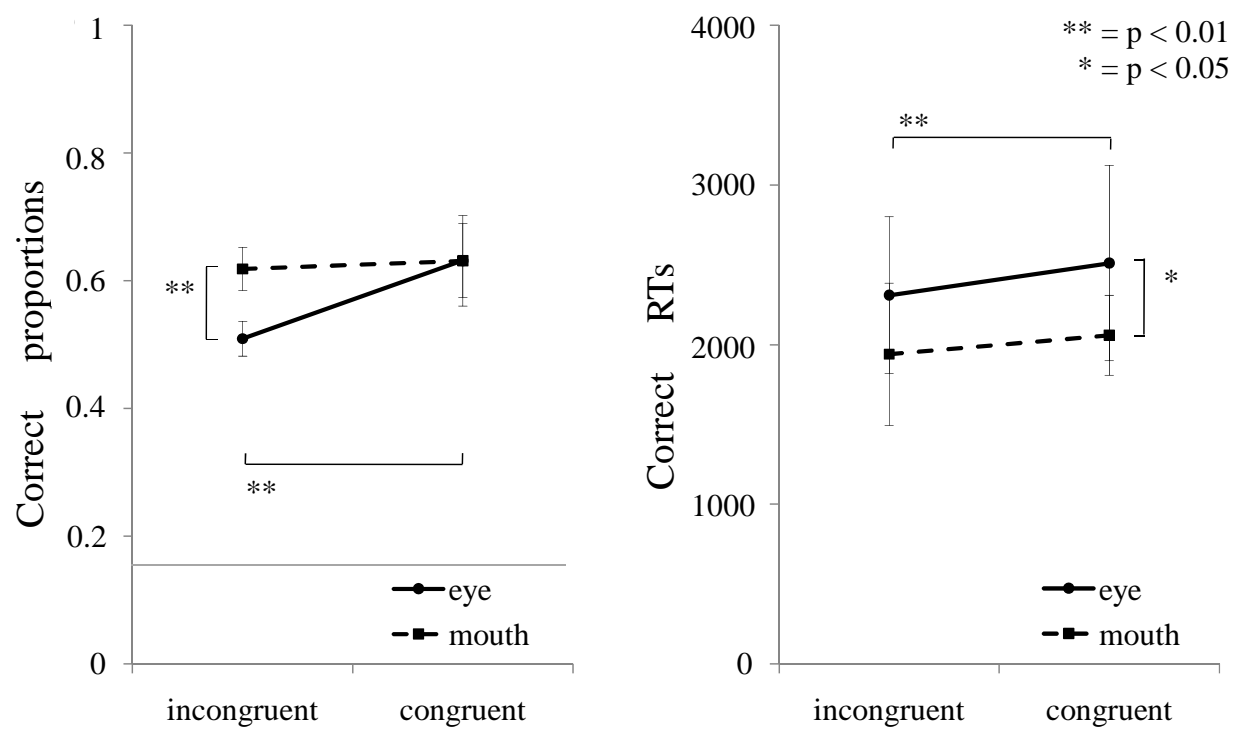

Figure 3. Mean correct proportions and RTs in Experiment 2. Error bars depict standard deviation. Overall, within the mouth-identification condition, there is no accuracy difference between incongruent and congruent condition. Within incongruent condition, eyes were more difficult to identify than the mouth. Moreover, there was no delay of RTs in incongruent condition.

This inversion effect was caused largely by disruptions in holistic processing. However, it is well-known that inversion disrupts other aspects of configural processing, such as second order relational processing, in addition to holistic processing (Maurer et al., 2002; Mondloch et al., 2002). Hence, the elimination of an accuracy difference based on congruence of inverted faces could also reflect the difficulty in detecting congruence differences due to a disruption of second-order relational processing. That is, it remained possible that in Experiment, the two second-order relational processing was disrupted in addition to a disruption of holistic processing. These two explanations could not be distinguished in Experiment 2. Therefore, in Experiment 3, we further explored whether second-order relational processing played a role in identifying single facial feature by using photographic negative images.

\section{Experiment 3}

Experiment 3 continued to employ the paradigm of Experiment 1, with the exception that the images were presented in a photographic negative transform. We investigated whether the role of configural processing of expression in identification of a single facial feature was resistant to the negation effect. Evidence of resistance to photo-negatives was diagnostic of the absence of second-order relational processing. If present, this resistance should manifest as lower accuracy and slower RTs for incongruent than congruent facial expressions, namely, a replication of findings in Experiment 1.

\section{Method}

Participants. Sixteen Japanese females aged between 22 and 40 years participated in the experiment for payment. All the participants were right-handed and had normal or corrected-to-normal vision.

Materials. The stimuli were the same as those in Experiment 1 except that the images were presented as 
photographic negatives.

Design. The design was identical to that of Experiment 1. This included repeated measures factors, stimulus congruence (incongruent and congruent) and task (eyes-identification and mouth-identification) resulting in the same four trial formats as in Experiments 1 and 2.

Procedure. The procedure was identical to that of Experiment 1.

\section{Results}

The participants' untransformed correct proportions and mean correct reaction times were summarized in Figure 4 (horizontal line indicates chance level responding).

Correct proportions. A two-factor repeated measures ANOVA investigating the same two factors defined in Experiment 1 (i.e., congruence and task) was performed on the arcsin-transformed correct proportions. There were significant main effects of stimulus congruence $\left(F_{(1,15)}=109.51, M S E=23.36, p<\right.$ $0.01)$ and task $\left(F_{(1,15)}=9.68, M S E=32.65, p<0.01\right)$, and a significant interaction between these factors $\left(F_{(1,15)}\right.$ $=98.83, M S E=6.67, p<0.01$ ) was observed. Further, post-hoc comparisons of performance on stimulus type within each task type showed significant differences ( $p<0.01$, Bonferroni corrected) between the incongruent and congruent conditions (incongruent corrects $<$ congruent corrects). Post-hoc comparisons of performance on tasks within each stimulus congruence level showed that there was a significant difference $(p<0.01$, Bonferroni corrected) between the eyes and mouth conditions only for the incongruent condition (eyes corrects $<$ mouth corrects).

Reaction times. A two-factor repeated measures ANOVA investigating the same two factors defined in Experiment 1 (i.e., congruence and task) was performed on the remaining mean correct RTs. There was no significant main effect of congruence $(F<1)$, but a significant main effect of task $\left(F_{(1,15)}=29.10, M S E=8.41\right.$, $p<0.01$; eyes RTs $>$ mouth RTs) was observed. Further, no significant interaction between stimulus type and task type $(F<1)$ was revealed.
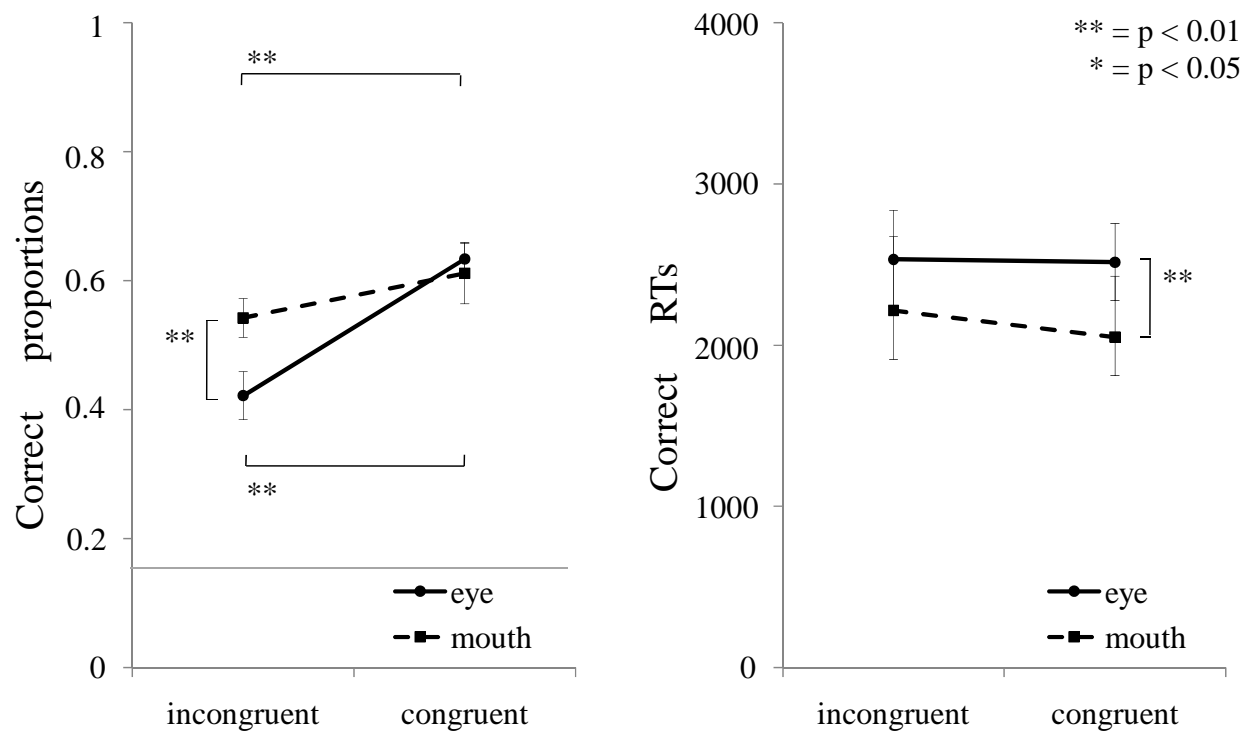

Figure 4. Mean correct proportions and RTs in Experiment 3. Error bars depict standard deviation. Overall, participants were significantly less accurate in identifying a single facial feature in the incongruent condition than in the congruent condition. Within incongruent condition, eyes were more difficult to identify than the mouth. Moreover, there was no difference of RTs between in incongruent condition and congruent condition. 


\section{Discussion}

The results of Experiment 3 almost replicated those of Experiment 1. This indicated that configural processing of expression played an important role in identification of a single facial feature, but that this processing did not include the second-order relational processing. Photo negation did not disrupt the role of configural processing of expression in identifying a single facial feature significantly. From this, it was possible to infer that the configural processing observed in Experiment 1 reflected only holistic processing, but not second-order relation processing.

Experiment 3 also confirmed that the strength of holistic configurational processing differed as a function of specific features. Within the incongruent condition, participants were significantly less accurate in identifying eyes than that in identifying mouth, whose result could reflect that eyes were less informative than the mouth.

\section{General Discussion}

This study had two goals. The first goal was to investigate whether configural processing of expression in recognizing facial expressions also affects identifications of the single facial features. The second goal was to ascertain if configural processing of facial expressions differentially affected the identification of specific facial features, namely the eyes and the mouth.

To address the first goal, we developed two different kinds of stimuli: Incongruent faces in which the single facial feature expressed an emotion that differed from the emotion expressed by the remaining parts of the face (e.g., happy eyes in a fearful face) and congruent faces in which the same emotion was expressed in the single feature and the rest of the face (e.g., happy eyes in a happy face). Experiment 1 found that the configural processing of expression played an important role in identifying a single facial feature. In the case of the incongruent condition, the participants were significantly less accurate in identifying the relevant (single) facial feature than that in the congruent condition. One interpretation of this effect was that configural processing of expression interacted with facial identity processing. Moreover, the results of Experiment 2 revealed that the incongruency effect disappeared when the faces were viewed upside down, at least for certain features (i.e., the mouth). However, in Experiment 3, no disruptions of the relevant configuration process were observed when the faces were viewed in photographic negatives. These results supported the idea that the kind of configural processing which played a role in the incongruency effect of Experiment 1 involved holistic processing, but not second-order relational processing. This is because holistic processing is impaired primarily by inversion whereas second-order relational processing is impaired by both inversion and negation. These findings concur with the previous studies showing a holistic processing for facial expressions (Calder et al., 2000; Calder \& Jansen, 2005).

The second goal focused upon the degree of holistic processing of emotion involved in identification specific features. Experiments 1, 2 and 3 showed that the strength of the expressive incongruence effect differed from that when the participants were asked to identify the isolated mouth to when they were asked to identify the isolated eyes. Contrary to our expectations, expressive eyes were more difficult to identify than an expressive mouth. This mouth dominance was quite strong as evident in its relative resistance not only to the upright (Experiment 1) but also to the inverse (Experiment 2) and the negative (Experiment 3). It is worth emphasizing that observations of mouth dominance are inconsistent with the idea that the eyes are more important than the mouth as claimed in folklore ("Eyes are more eloquent than lips") and the fact that other 
studies have shown a distinct speciality of the eyes (Baron-Cohen, 1995; Baron-Cohen et al., 1997). This recessiveness of the eyes could suggest that eyes are more susceptible to holistic processing than mouth. Eyes may not be more eloquent than lips. One possible interpretation for the finding that eyes appear less informative than anticipated is that there is difference in size of the facial feature between the eyes and the mouth.

However, the current consensus in the research on facial identity regards eye-region information as dominant over that of the mouth. Faces were more readily recognized from the eye region than the mouth region (Ellis, Shepherd, \& Davies, 1979; McKelvie, 1976; Shepherd, Davies, \& Ellis, 1981; Young, Hay, McWeeny, Flude, \& Ellis, 1985). Yarbus (1967) also showed that gaze fixations are longer when the object of a viewer's gaze contained more information concentrating on the eyes in a face. This would imply that the eyes play a special role in facial processing. Then, the question of whether eyes are truly not special in any other aspect of facial processing, such as recognition of emotional expressions remains. Although this issue has not been the focus of the present study, it is essential that it should be addressed in future studies.

\section{References}

Baron-Cohen, S. (1995). Mind blindness: An essay on autism and theory of mind. Cambridge, M. A.: MIT Press

Baron-Cohen, S., Wheelwright, S., \& Joliffe, A. T. (1997). Is there a language of the eyes? Evidence from normal adults and adults with autism or Asperger syndrome. Visual Cognition, 4, 311-331.

Barton, J. J. S., Depak, S., \& Malik, N. (2003). Attending to faces: Change detection, familiarization, and inversion effects. Perception, 32, 15-28.

Barton, J. J. S., Keenan, J. P., \& Bass, T. (2001). Discrimination of spatial relations and features in faces: Effects of inversion and viewing duration. British Journal of Psychology, 92, 527-549.

Calder, A. J., \& Jansen, J. (2005) Configural coding of facial expressions: The impact of inversion and photographic negative. Visual Cognition, 12, 495-518.

Calder, A. J., Young, A. W., Keane, J., \& Dean, M. (2000). Configural information in facial expression perception. Journal of Experimental Psychology: Human Perception and Performance, 26, 527-551.

Carey, S., \& Diamond, R. (1994). Are faces perceived as configurations more by adults than by children? Visual Cognition, 1, 253-274.

Collishaw, S. M., \& Hole, G. J. (2000). Featural and configurational processes in the recognition of faces of different familiarity. Perception, 29, 893-909.

Durand, K., Gallay, M., Seigneuri, A., Robichon, F., \& Baudouin, J. (2007). The development of facial emotion recognition: The role of configural information. Journal of Experimental Child Psychology, 97, 14-27.

Ellis, H. D., Shepherd, J. W., \& Davies, G. M. (1979). Identification of familiar and unfamiliar faces from internal and external features: Some implications for theories of face recognition. Perception, 8, 431-439.

Fallshore, M., \& Bartholow, J. R. (2003). Recognition of emotion from inverted schematic drawings of faces. Perceptual and Motor Skills, 96, 236-244.

Farah, M. J., Tanaka, J. W., \& Drain, H. M. (1995). What causes the face inversion effect? Journal of Experimental Psychology: Human Perception and Performance, 21, 628-634.

Freire, A., \& Lee, K. (2001). Face recognition in 4 to 7 year olds: Processing of configural, featural, and paraphernalia information. Journal of Experimental Child Psychology, 80, 347-371.

Freire, A., Lee, K., \& Symons, L. A. (2000). The face-inversion effect as a deficit in the encoding of configural information: Direct evidence. Perception, 29, 159-170.

Gallegos, D. R., \& Tranel, D. (2005). Positive facial affect facilitates the identification of famous faces. Brain \& Language, 93, 338-348.

Galper, R. E. (1970). Recognition of faces in photographic negatives. Psychonomic Science, 19, 207-208.

Galper, R. E., \& Hochberg, J. (1971). Recognition memory for photographs of faces. American Journal of Psychology, 84, 351-354.

Ganel, T., Valyear, K. F., Goshen-Gottstein, Y., \& Goodale, M. A. (2005). The involvement of the "fusiform face area" in processing facial expression. Neuropsychologica, 43, 1645-1654. 
Hayes, T., Morrone, M. C., \& Burr, D. C. (1986). Recognition of positive and negative band pass-filtered images. Perception, 15, 595- 602.

Hole, G. I. (1994). Configural factors in the perception of unfamiliar faces. Perception, 23, 65-74.

Hole, G. I., George, P. A., \& Dunsmore, V. (1999). Evidence for holistic processing of faces viewed as photographie negatives. Perception, 28, 341-359.

Kaufmann, J. M., \& Schweinberger, S. (2007). Expression influences the recognition of familiar faces. Perception, 33, $399-408$.

Kemp, R., McManus, C., \& Pigott, T. (1990). Sensitivity to the displacement of facial features in negative and inverted images. Perception, 19, 531-543.

Kemp, R., Pike, G., White, P., \& Musselman, A. (1996). Perception and recognition of normal and negative faces: The role of shape from shading and pigmentation cues. Perception, 25, 37-52.

Lander, K., \& Metcalfe, S. (2007). The influence of positive and negative facial expressions on face familiarity. Memory, 15, 63-69.

Leder, H., \& Bruce, V. (1998). Local and relational aspects of face distinctiveness. Quarterly Journal of Experimental Psychology, $51,449-473$.

Leder, H., \& Bruce, V. (2000). When inverted faces are recognized: The role of configural information in face recognition. Quarterly Journal of Experimental Psychology, 53, 513-536.

Leder, H., Candrian, G., Huber, O., \& Bruce, V. (2001). Configural features in the context of upright and inverted faces. Perception, 30, 73-83.

Le Grand, R., Mondloch, C. J., Maurer, D., \& Brent, H. P. (2001). Early visual experience and face processing. Nature, 410, 890.

Le Grand, R., Mondloch, C. J., Maurer, D., \& Brent, H. P. (2004). Impairment in holistic face processing following early visual deprivation. Psychological Science, 15, 762-768.

Lewis, M. B., \& Johnston, R. A. (1997). The Thatcher Illusion as a test of configural disruption. Perception, 26, 225-227.

Maurer, D., Le Grand, R., \& Mondloch, C. J. (2002). The many faces of configural processing. Trends in Cognitive Sciences, 6 , 255-260.

McKelvie, S. J. (1976). The role of eyes and mouth in the memory of a face. American Journal of Psychology, 89, 311-323.

Michel, C., Rossion, R., Han, J., Chung, C., \& Caldara, R. (2006). Holistic processing is finely tuned for faces of one's own race. Psychological Science, 17, 608-615.

Mondloch, C. J., Le Grand, R., \& Maurer, D. (2002). Configural face processing develops more slowly than featural face processing. Perception, 31, 553-566.

Posamentier, M. T., \& Abdi, H. (2003). Processing faces and facial expressions. Neuropsychology Review, 13, 113-143.

Rhodes, G., Brake, S., \& Atkinson, A. P. (1993). What's lost in inverted faces? Cognition, 47, 25-57.

Schweinberger, S. R., \& Soukup, G. R. (1998). Asymmetrical relationships among perceptions of facial identity, emotion and facial speech. Journal of Experimental Psychology-Human Perception and Performance, 24, 1748-1765.

Searcy, J. H., \& Bartlett, J. C. (1996). Inversion and processing of component and spatial-relational information in faces. Journal of Experimental Psychology: Human Perception and Performance, 22, 904-915.

Shepherd, J. W., Davies, G. M., \& Ellis, H. D. (1981). Studies of cue saliency. In G. M. Davies, H. D. Ellis, \& J. W. Shepherd (Eds.), Perceiving and remembering faces (pp. 105-131). London: Academic Press.

Tanaka, J. W., Kay, J. B., Grinnell, E., Stansfield, B., \& Szechter, L. (1998). Face recognition in young children: When the whole is greater than the sum of its parts. Visual Cognition, 5, 479-496.

Tanaka, J. W., \& Farah, M. (1993). Parts and wholes in face recognition. Quarterly Journal of Experimental Psychology, 46, 225-245.

Tanaka, J. W., \& Sengco, J. A. (1997). Features and their configuration in face recognition. Memory and Cognition, $25,583-592$.

Yarbus, A. L. (1967). Eye movements and vision. New York: Plenum.

Young, A. W., Hay, D. C., McWeeny, K. H., Flude, B. M., \& Ellis, A. W. (1985). Matching familiar and unfamiliar faces on internal and external features. Perception, 14, 737-746.

Young, A. W., Hellawell, D., \& Hay, D. C. (1987). Configurational information in face perception. Perception, 16, 747-759.

Yin, R. K. (1969). Looking at upside-down faces. Journal of Experimental Psychology, 81, 141-145.

White, M. (2000). Parts and wholes in expression recognition. Cognition and Emotion, 14, 39-60.

White, M. (2001). Effect of photographic negation on matching the expressions and identities of faces. Perception, 30, 969-981.

Wild-Wall, N., Dimigen, O., \& Sommer, W. (2008). Interaction of facial expressions and familiarity: ERP evidence. Biological Psychology, 77, 138-149. 